\title{
A resonating rainfall and evaporation recorder
}

\author{
Ryan D. Stewart, ${ }^{1}$ Rolf Hut, ${ }^{2}$ David E. Rupp, ${ }^{3}$ Hoshin Gupta, ${ }^{4}$ and John S. Selker ${ }^{1}$ \\ Received 24 October 2011; revised 12 June 2012; accepted 13 June 2012; published 14 August 2012. \\ [1] We propose a novel, accurate quantification of precipitation and evaporation, as needed \\ to understand fundamental hydrologic processes. Our system uses a collection vessel placed \\ on top of a slender rod that is securely fixed at its base. As the vessel is deflected, either \\ by manual perturbation or ambient forcing (for example, wind), its oscillatory response \\ is measured, here by a miniature accelerometer. This response can be modeled as a damped \\ mass-spring system. As the mass of water within the collection vessel changes, either \\ through the addition of precipitation or by evaporative loss, the resonant frequency \\ experiences an inverse shift. This shift can be measured and used to estimate the change in \\ the mass of water. We tested this concept by creating a simple prototype which was used \\ in field conditions for a period of 1 month. The instrument was able to detect changes \\ in mass due to precipitation with an accuracy of approximately $1 \mathrm{~mm}$.
}

Citation: Stewart, R. D., R. Hut, D. E. Rupp, H. Gupta, and J. S. Selker (2012), A resonating rainfall and evaporation recorder, Water Resour. Res., 48, W08601, doi:10.1029/2011WR011529.

\section{Introduction}

[2] Accurate measurement of precipitation and evaporation is crucial to the understanding of fundamental hydrologic processes, but achieving reliable estimates often involves a tradeoff between accuracy, dependability and cost.

[3] Instruments that measure precipitation rates directly (i.e., nonremotely) include tipping buckets, weighing gauges, optical sensors, capacitance sensors, acoustical sensors, and disdrometers, with tipping buckets and weighing gauges being the most common [Nystuen, 1999; Duchon and Essenberg, 2001]. Weighing gauges use a scale to calculate the weight of water in a collection vessel; types of scales used include counterbalance weights, springs, and strain gauges. In the case of a spring-based weighing platform, the deflection can be sensed using potentiometers or linear variable differential transformers [Tanner, 1990], or a chart recorder, such as traditionally used by the U.S. National Weather Service (NWS) [Kuligowski, 1997]. More recently, the "GeoNor" gauge scale was developed that suspends a collection vessel from one or more wires and calculates the weight of water from the tension of one wire, determined by vibrating the wire and measuring its resonant frequency [Bakkehøi et al., 1985; Duchon, 2008]. Another meteorological sensor based on vibration frequency is the icing sensor used by the NWS in its Automated Surface Observing

\footnotetext{
${ }^{1}$ Department of Biological and Ecological Engineering, Oregon State University, Corvallis, Oregon, USA.

${ }^{2}$ Department of Water Management, Faculty of Civil Engineering and Geosciences, Delft University of Technology, Delft, Netherlands.

${ }^{3}$ Oregon Climate Change Research Institute, College of Earth, Ocean and Atmospheric Sciences, Oregon State University, Corvallis, Oregon, USA.

${ }^{4}$ Department of Hydrology and Water Resources, University of Arizona, Tucson, Arizona, USA.
}

Corresponding author: R. D. Stewart, Department of Biological and Ecological Engineering, Oregon State University, Corvallis, Oregon, USA. (stewarry@onid.orst.edu)

(C) 2012. American Geophysical Union. All Rights Reserved. 0043-1397/12/2011WR011529
System (ASOS). Freezing rain and ice accumulations are inferred by measuring the vibration frequency of a small cylindrical probe; the frequency changes as mass accumulates on the probe [Tattelman, 1982].

[4] Each system is subject to different limitations. Many disdrometers (such as impact disdrometers) tend to underestimate the rainfall rate due to insensitivity to small droplets, while acoustic methods tend to have higher variability and greater bias than other methods [Nystuen, 1999]. Optical rain gauges only measure rainfall rate, not total accumulation, and therefore have inherent uncertainty due to the need to estimate drop size distributions [Nystuen, 1999]. Capacitance gauges have been seen to give good results, but have relatively high noise when the rainfall rate is low (less than $2 \mathrm{~mm} \mathrm{~h}^{-1}$ ), and have increased scatter in windy conditions [Nystuen, 1999]. Tipping buckets can have significant error at small and large rainfall rates, can fail to tip due to blockage or friction, will have different tipping volumes at different rainfall intensities, and can lose water to evaporation between tips [Nystuen et al., 1996; Humphrey et al., 1997; Nystuen, 1999; Upton and Rahimi, 2003]. Weighing buckets may underreport data due to friction in the bearings and potentiometer linkage [Duchon and Essenberg, 2001], and can be affected by temperature- and wind-induced noise [Nayak et al., 2008]. In addition, weighing buckets must be periodically emptied, either manually or through an autosiphon, leading to error whenever the bucket overtops or drains during a rainfall event [Nayak et al., 2008] or when the drainage system fails completely [Nystuen, 1999]. Cablebased weighing gauges have higher noise as wind and/or collected mass increase [Duchon, 2008]. The ASOS icing sensors only measure accumulated frozen precipitation, and thus are not useful for measuring liquid or solid precipitation. Finally, all of these methods, with the exceptions of optical sensors, acoustic sensors and disdrometers, are generally unusable for monitoring rainfall on ocean-placed buoys or floating platforms [Nystuen et al., 1996].

[5] Instruments that directly measure evaporation are relatively rare compared to those used to measure precipitation. 
Often, reference evaporation is estimated from predictive equations based on meteorological parameters, with a large variation in results between models [Grismer et al., 2002], or else estimated from indirect measurements such as infrared surface temperatures [Nemani and Running, 1989] or water vapor and nitrogen gas concentrations [Drexler et al., 2004]. Less commonly, evaporation is determined using a water balance approach, such as pan evaporation [Grismer et al., 2002] or weighing and/or small-scale lysimeters [Dugas and Bland, 1989]. All of these methods require extensive effort and/or expense to implement, and are therefore not practical for small-scale farmers and other irrigators. The ETGage Company (Loveland, CO) sells an evapotranspiration simulator that allows water to evaporate through a clayceramic plate. However, to be automated, this instrument requires the purchase of an external data logger; further, the instrument needs to be regularly refilled with water, and the ceramic disc can become fouled by minerals if it is contacted by irrigation water or precipitation.

[6] In this note, we propose a simple method that uses the natural frequency of the gauge itself to measure the mass of the system (thereby allowing inference of cumulative precipitation and evaporation amounts), and show preliminary results from a prototype configuration. While resonance of tensioned load wires has long been used to measure the accumulation of certain types of precipitation, including snowfall (with the GeoNor system) and freezing rain (with the ASOS icing instrument), our method can be configured to measure both precipitation (in liquid or solid form) and evaporation. Furthermore, the instrumentation needed is relatively inexpensive, contains no moving parts, uses lowpower solid state microelectronics, can be easily calibrated, and can theoretically be designed to be insensitive to wind or buffeting. Unlike other precipitation measurement sensors, our configuration has the potential to take advantage of ambient driving forces such as winds or waves, and can therefore be placed in remote or challenging environments. Altogether, simplicity of this method's design, components and calibration should enable wide use by researchers as well as irrigation and urban-planning managers.

\section{Materials and Methods}

[7] At its most basic configuration our rain gauge system is a damped mass-spring system, where the collection bucket is the mass and a flexible pole acts as the spring. If the bucket is displaced, through either manual/mechanical or natural forcing, the natural frequency of its response will be a function of the mass of the bucket.

[8] When a damped mass-spring system is displaced, its position through time can be modeled as

$$
z(t)=A e^{-\zeta \omega_{0} t} \sin \left(\sqrt{1-\zeta^{2}} \omega_{0} t+\varphi\right)
$$

where $z(t)$ is the deviation from resting position, $A$ is the amplitude of the impulse, $\varphi$ is an initial phase, $\zeta$ is the damping ratio, and $\omega_{0}$ is the undamped natural frequency.

[9] The damping ratio, $\zeta$, is given by

$$
\zeta=\frac{c}{2 m \omega_{0}}
$$

where $c$ is the viscous damping coefficient and $m$ is the mass.
[10] The undamped natural frequency, $\omega_{0}$, is given (in $\mathrm{rad} / \mathrm{s}$ ) by

$$
\omega_{0}=\sqrt{\frac{k}{m}}
$$

where $\mathrm{k}$ is the spring stiffness.

[11] It should be noted that the observed frequency of oscillation $\left(\omega_{1}\right)$ is slightly lower than the undamped natural frequency, as shown by

$$
\omega_{1}=\omega_{0} \sqrt{1-\zeta^{2}}
$$

Therefore, in the case of known, manual forcing (such as during calibration), equation (1) can be fitted to the observed oscillation, using $A, \zeta, \omega_{0}$, and $\varphi$ as parameters. This is called time domain fitting. In the case of ambient forcing (such as in the case of wind), the measured signal is a convolution of equation (1) with the (unknown) ambient force. The resulting time series cannot be fitted to a known form. However, the frequency spectrum of wind has been shown to be a power law function [Hwang, 1970]. The total frequency spectrum of the measured signal is the multiplication of the frequency spectrum of the wind (forcing) with the frequency power spectrum of the impulse response (i.e., equation (1)) [Lathy, 1998]. The amplitude of the frequency spectrum of the impulse response, $|Z(\omega)|$, is derived by taking the Fourier transform of equation (1):

$$
|Z(\omega)|=\frac{1}{\sqrt{\omega_{0}^{2}-2 \zeta^{2}-\omega^{2}}+4 \zeta^{2}\left(\omega_{0}^{2}-\zeta^{2}\right)}
$$

leading to an expected amplitude of the power spectrum of the measured signal, $|H(\omega)|$ :

$$
|H(\omega)|=\omega^{\alpha} \frac{1}{\sqrt{\omega_{0}^{2}-2 \zeta^{2}-\omega^{2}}+4 \zeta^{2}\left(\omega_{0}^{2}-\zeta^{2}\right)}
$$

where $\omega^{\alpha}$ is the frequency (power) spectrum of the driving wind force. This equation can be fitted to the frequency spectrum of measured data, again using $\omega_{0}, \zeta$, and $\alpha$ as parameters. The fitting procedure must be designed in such a way that $\omega_{0}$ is the most sensitive parameter. To achieve this, fitting is only done on a window of the frequency spectrum around $\omega=\omega_{0}$, making sure that a deviation in $\omega_{0}$ is weighted more strongly than deviations in the other parameters. The choice of window is arbitrary, so long as the window is sufficiently large to include the expected minimum and maximum resonance frequencies. These minimum and maximum frequencies can be found by doing a controlled experiment, i.e., during calibration. The relation between the resonance frequency and the mass of the amount of water in the gauge, and thus the cumulative rainfall, is also obtained by doing a controlled experiment, resulting in a calibration curve. It should also be noted that $0.001 \mathrm{~g}$ was set as the minimum threshold for signal amplitude; windows whose maximum amplitude fell below that threshold were not included in the output.

[12] A prototype system (Figure 1) was constructed using $0.013 \mathrm{~m} \times 0.0035 \mathrm{~m}$ steel strap for the system's spring. An asymmetrical spring was chosen to ensure distinct and separate resonant modes between the main and transverse 


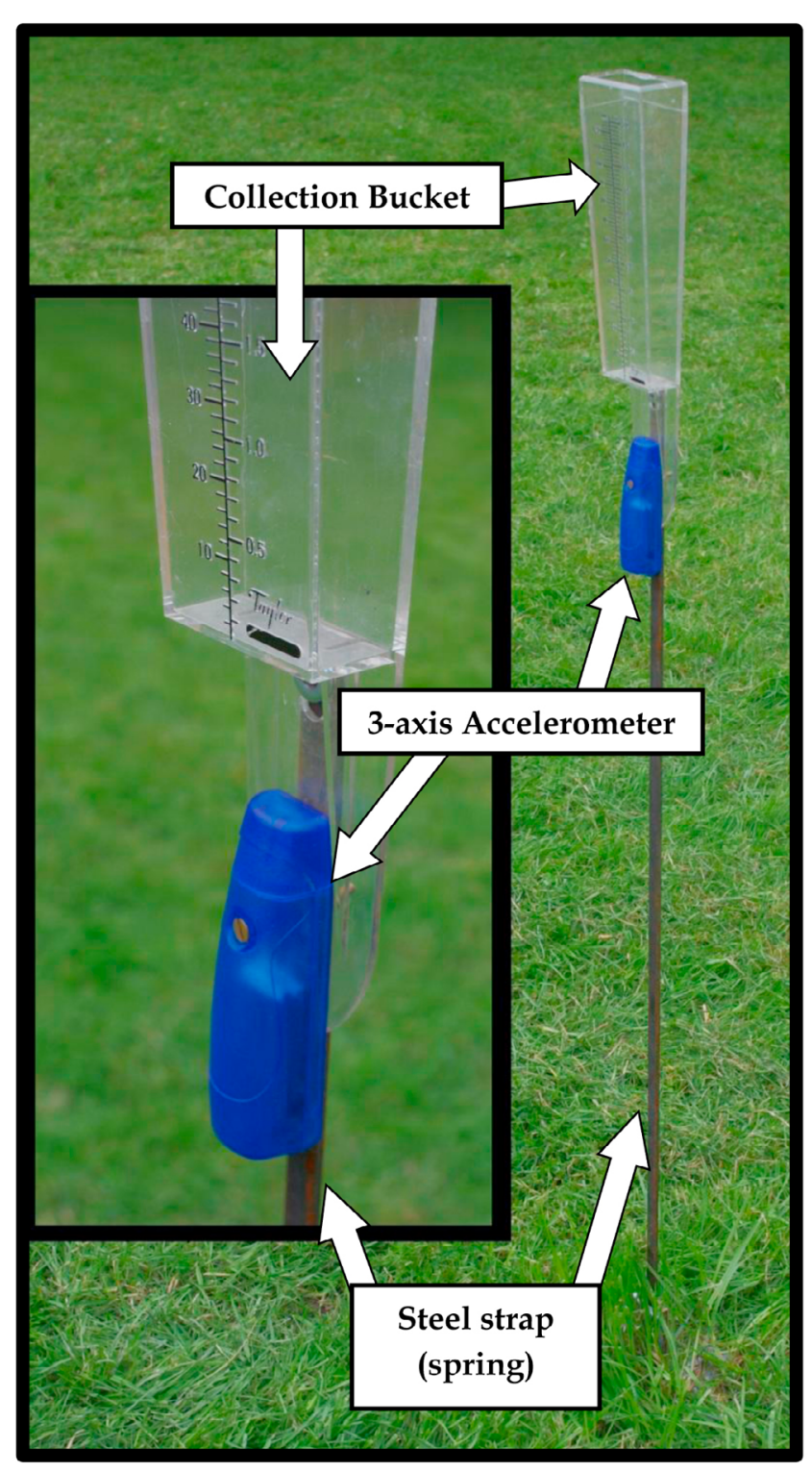

Figure 1. Major components of the precipitation and evaporation gauge prototype (inset is a close-up of the accelerometer and collection bucket). The collection bucket acts as the mass, and the steel strap acts as a spring in a simple damped mass-spring system. Oscillations of the mass-spring system (after lateral deflection) are measured using the three-axis accelerometer. For reference, the accelerometer is $10 \mathrm{~cm}$ in length.

directions. This strap was attached to a heavy four-legged steel base using two $0.001 \mathrm{~m}(0.375 \mathrm{inch})$ bolts; this base had a $0.001 \mathrm{~m} \times 0.0635 \mathrm{~m}(0.375 \times 2.5 \mathrm{inch})$ bolt at the end of each leg, which allowed the base to be securely set into the soil. The strap's effective length (measured from the uppermost fastening bolt to the base of the collection bucket) was $0.87 \mathrm{~m}$. A clear plastic rain gauge (Taylor Precision model $2702 \mathrm{~N}$ ) was used as the collection bucket, as this allowed for visual confirmation of the amount of water in the gauge. This gauge was attached to the strap using two \#8 $(0.0033 \mathrm{~m})$ bolts; the lower bolt also served to attach a three-axis 16-bit accelerometer (Model X6-2 - Gulf Coast Data Concepts, LLC). The accelerations of the gauge were measured at a sampling frequency of $80 \mathrm{~Hz}$. For the outdoor testing, the accelerometer was placed within a sealed $0.5 \mathrm{~L}$ plastic bag before being secured to the system.

[13] On 10 March 2012, the prototype was installed in an open outdoor location in Corvallis, Oregon, United States; data collection began the following day. Data collection was continuous except for when the accelerometer was removed to allow for the battery to be recharged for 4 to $8 \mathrm{~h}$. No data were collected between 18 March 2012 and 27 March 2012. To verify the instrument's accuracy, visual readings of the amount of water within the rain gauge collection's bucket were made between 1 and 4 times per day. When the amount of water within the gauge exceeded $90 \mathrm{~mm}$, the gauge was unfastened and pivoted to empty. The data collection period included two major storm systems. Wind speeds, monitored by an adjacent Memsic Inc. (formerly Crossbow) ES2000 Weather Station Sensor Suite weather station, reached a maximum of $18.6 \mathrm{~km} \mathrm{~h}^{-1}$, with a mean maximum speed of $5.9 \mathrm{~km} \mathrm{~h}^{-1}$

[14] On 13 April 2012, the prototype was calibrated in situ by adding known depths of water to the collection bucket. The following depths (in millimeters) of water were tested: $0,10,20,30,40,50,60,70,80,90$, and 100. For each measurement, the bucket was manually displaced by approximately $0.10 \mathrm{~m}$ and released, and the resulting oscillation was recorded by the accelerometer. After being manually displaced, the gauge was then allowed to collect data for at least an additional ten minutes, to allow for the ambient forcing to occur. This combination of manual displacement and natural forcing allowed for calibration in both the time and frequency domains.

[15] To interpret the physically displaced (oscillatory) data, the relationship between natural frequency and mass was derived by fitting equation (1). Time domain fitting was performed by manually identifying the beginning (just after the instrument was displaced and released) and then performing the analysis over the next $25 \mathrm{~s}$. The resultant oscillatory data were modeled by optimizing for $A, \zeta, \omega_{0}$, and $\varphi$, using the Matlab function nlinfit [Holland and Welsch, 1977]. Because optimization using nlinfit minimizes RMSE residuals, only the portion of the spectrum immediately around $\omega_{0}$ was used. This ensured that no weight was placed on the very low frequency portion of the spectra, which (due to wind influences) typically has a much higher amplitude. An example data set and model in the time domain are presented in Figure 2 (top), which shows the model is correctly capturing the system's frequency and damping.

[16] The ambient forcing calibration data were modeled in the frequency domain using equation (6). To fit data in the frequency domain, one must decide on the duration of signal to be used to represent one time step. A larger interval of data collection will contain more data points, thereby allowing for higher accuracy in the determination of the resonance frequency, whereas a smaller window allows for better time resolution. If the chosen data window is too long, the resonance frequency could shift during the interval (due to rain or evaporation); however, the window should be selected to be long enough for the resonance frequency to be observable within each windowed data set. The decision was made to use a 10 min window (containing 48,000 data points); this length of window provided ample data to allow accurate estimation of the resonance frequency, while being short enough that changes in mass due to precipitation or 

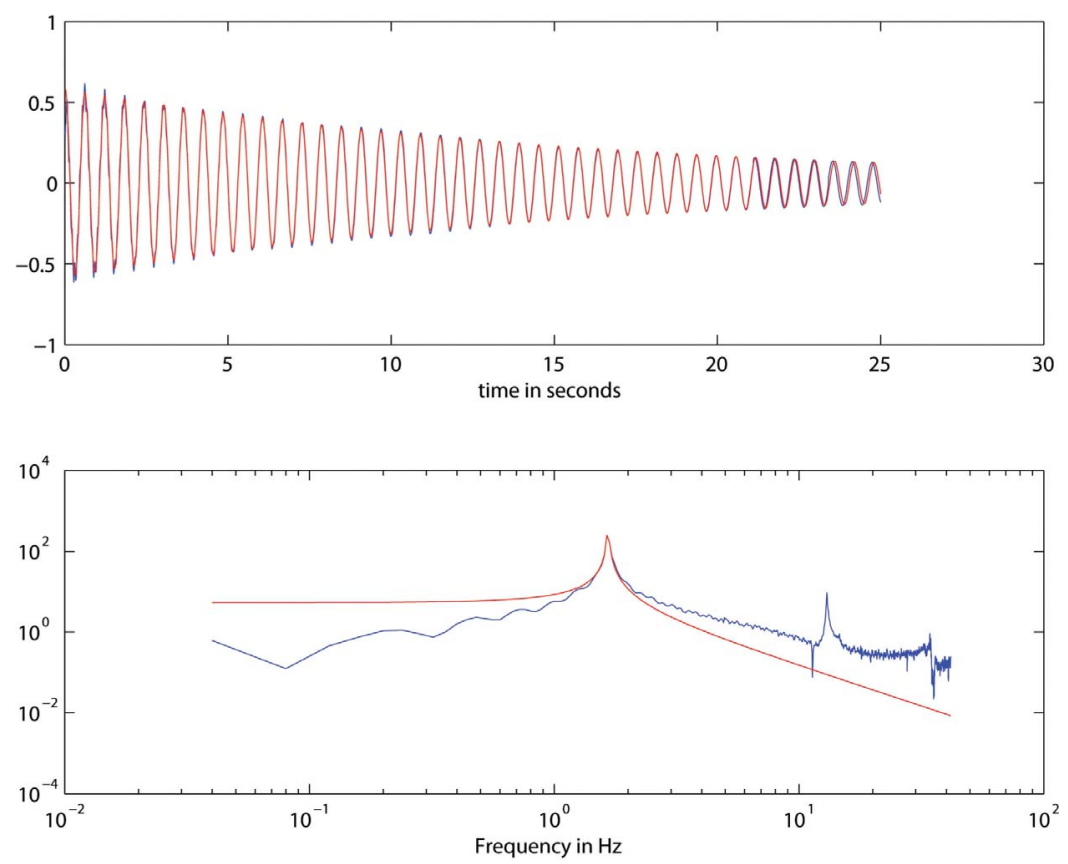

Figure 2. Acceleration response of the system after being manually deflected and released. Data are shown in both the (top) time and (bottom) frequency domains. Measured data are shown in blue, while modeled fit is shown in red. There is a clear resonant peak near $1.7 \mathrm{~Hz}$ due to the main oscillatory axis, whereas the secondary peak at $12 \mathrm{~Hz}$ corresponds to vibrations in the transverse axis. For this reason, it is important to focus the fitting algorithm around the expected frequency range. Also note that ambient forcing data can only be modeled in the frequency domain.

evaporation would not cause a significant shift in resonant frequency. An example of data fit in the frequency domain is shown in Figure 2 (bottom). The highest peak, around $1.7 \mathrm{~Hz}$, is the resonant frequency of the main axis, whereas the secondary peak around $12 \mathrm{~Hz}$ is due to vibrations in the transverse axis. Theoretically, with proper calibration, either mode could be used for the analysis, but the decision was made to focus on the lower resonant peak, which corresponds to the resonance of the main axis.

\section{Results and Discussion}

[17] Our experiments with the prototype show that an inverse relationship exists between undamped frequency and depth of water in the collection bucket (Figure 3). For this proof of concept a linear approximation (shown as the linear fit line) was deemed acceptable, though certainly a higher-order curve fit could be utilized, with a corresponding improvement to the accuracy. Figure 3 shows that the time and frequency domain models give nearly identical results, and thus either is a feasible method of determining system resonant frequency.

[18] When left outside during periods of intense rainfall and moderate winds, the instrument was able to accurately capture changes in mass of the system (through additions of precipitation and losses due to emptying or adjusting the gauge; Figures 4 and 5). The measured data correspond very well to the data obtained through visual checks of the water level within the gauge (shown in red in Figures 4 and 5), with an accuracy of approximately $\pm 1 \mathrm{~mm}$ through much of the range (the exact accuracy is difficult to quantify due to the low resolution of the gauge's visual markings). The variability of adjacent data points during nonrainy periods gives an indication of the instrument's precision: maximum variability was observed to be $\pm 1 \mathrm{~mm}$. In both Figures 4 and 5 , negative water amounts (down to $-5 \mathrm{~mm}$ ) were measured when the gauge is empty, due to the linear calibration used. As seen in Figure 3, the linear calibration curve has its greatest error when the gauge is empty; using a nonlinear fit should reduce or alleviate this bias.

[19] Even though this prototype was configured for precipitation collection, evaporation measurements could be obtained with the same gauge because the instrument is measuring the mass of the system (evaporation data from a separate prototype are presented in the auxiliary material). ${ }^{1}$ While this is theoretically possible in any weighing gauge system, there have been few or no rain gauges that have been configured to allow and measure evaporation.

[20] In addition, these data show that wind can be a satisfactory forcing mechanism for the system. Using wind as the driving force has several advantages compared to mechanical activation: it is simple to implement, and data is continuously collected. Moreover, with modern, low-cost, high-sensitivity accelerometers, wind-forced gauges have the potential to be designed in a way to directly measure wind speed and direction, and thereby provide a potential alternative to an anemometer for the purpose of making wind corrections to collected rainfall [e.g., Sieck et al., 2007]. However, there are also several minor disadvantages to relying only on wind to drive the sensor. First, in most locations, wind is unpredictable and readings may not be obtainable during very calm

\footnotetext{
${ }^{1}$ Auxiliary materials are available in the HTML. doi:10.1029/ 2011WR011529.
} 


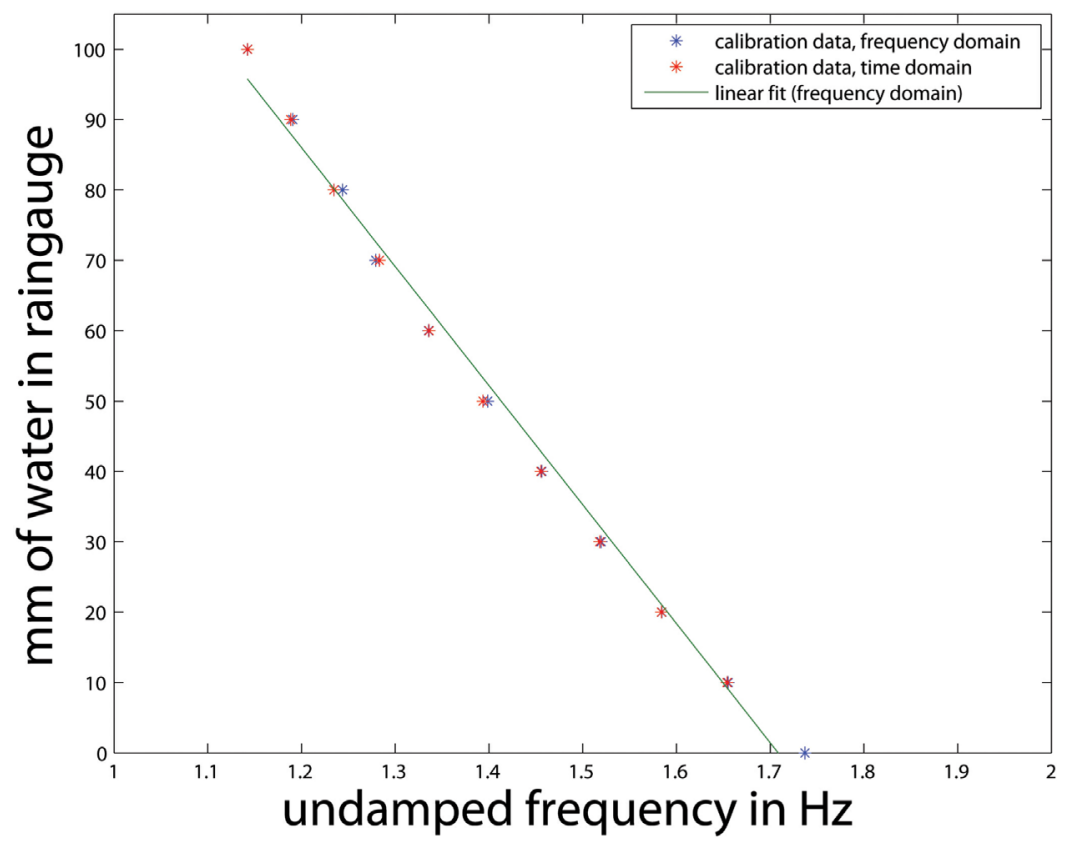

Figure 3. Undamped frequency of the prototype rainfall measurement system versus depth of water in the rain gauge (in millimeters). The plot indicates a nonlinear inverse relationship between undamped frequency and depth of water. The linear fit represents the calibration curve used to convert frequency into equivalent water depth within the rain gauge.

periods (during data collection, we observed a few isolated periods when the device was not accelerated past our imposed $0.001 \mathrm{~g}$ amplitude threshold), although with higherresolution accelerometers and better data processing we anticipate the minimum signal threshold would decrease markedly. Second, the acceleration magnitudes are typically smaller and the signals are affected by both the energy spectrum of the natural forcing and the properties of the device, in contrast to a mechanically vibrated system for which a simple impulse response is obtained. Third, the acceleration data from a naturally forced system must be analyzed by averaging over specified time intervals

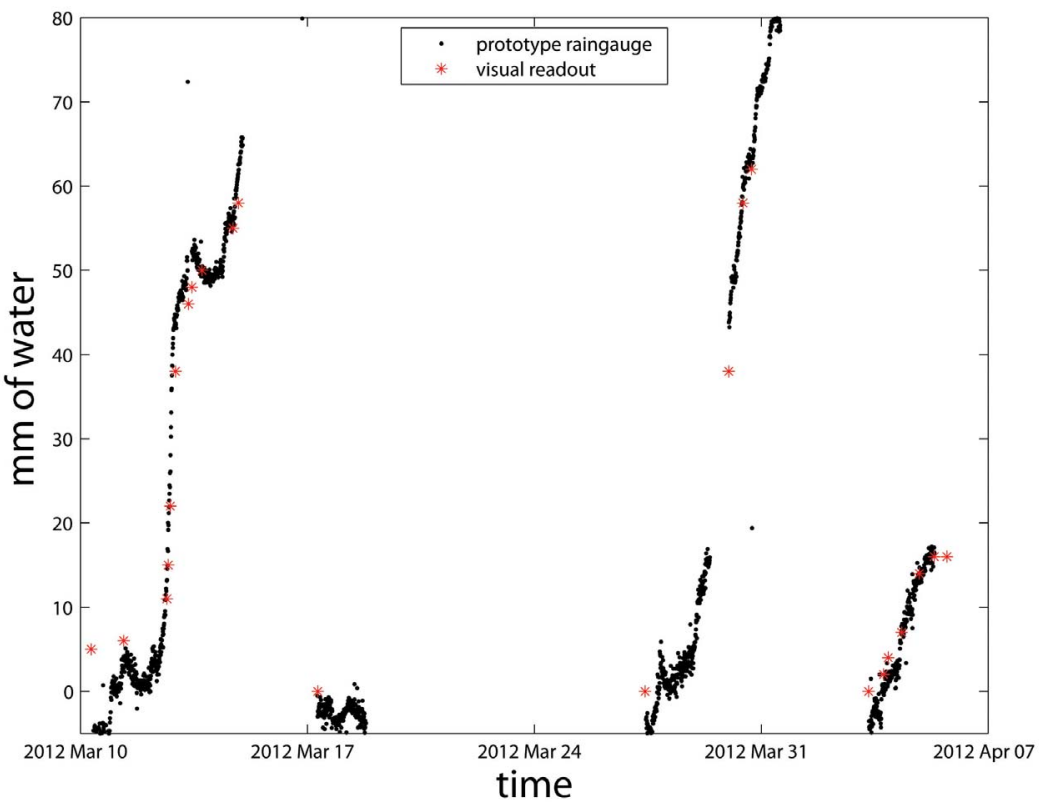

Figure 4. Measured precipitation accumulation within the prototype gauge, along with visual checks of the amount of water within the rain gauge. The amount of water within the gauge was inferred by determining the system's undamped frequency by frequency domain fitting of the acceleration data over $10 \mathrm{~min}$ analysis windows. Data gaps correspond to times when the accelerometer either ran out of power or was removed from the gauge for recharging. 

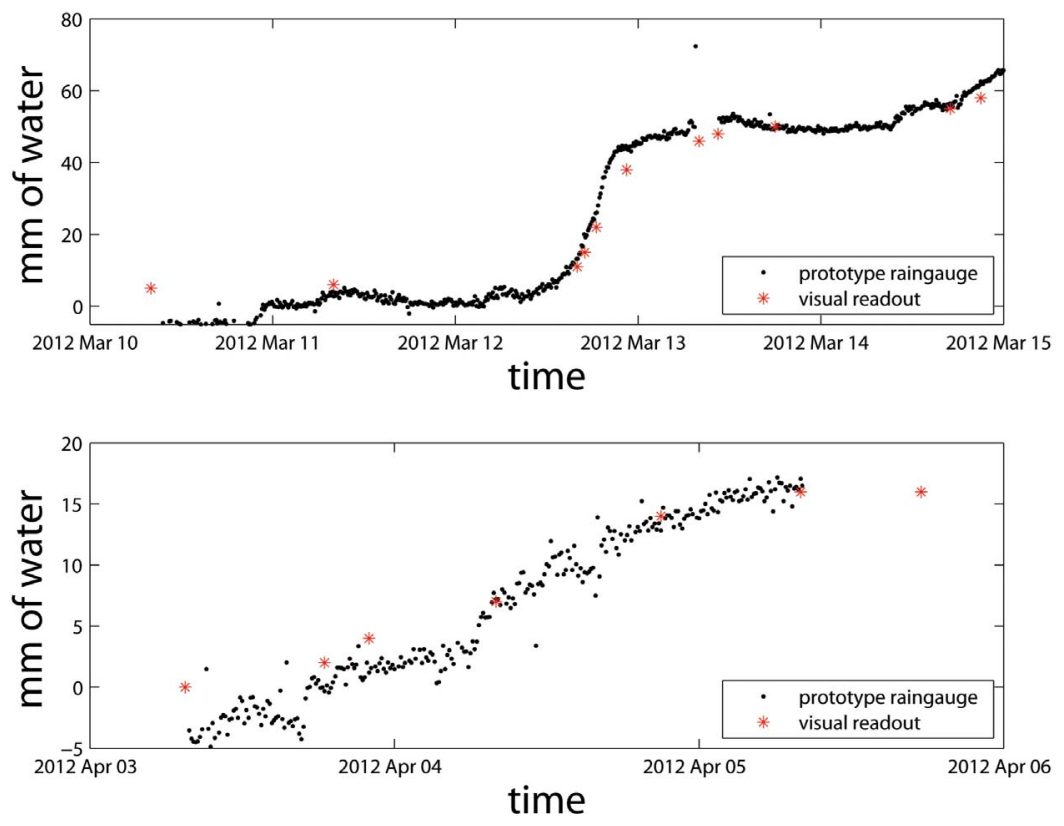

Figure 5. Close focus on the (top) first and (bottom) third rainfall events. These plots show that the prototype configuration, when subjected only to natural forcing from the wind, has an accuracy of approximately $\pm 1 \mathrm{~mm}$ (based on the comparison to visually measured amounts of water in the gauge) and precision of approximately $\pm 1 \mathrm{~mm}$ (based on the variability of consecutive measurements under no rain conditions). Data gaps correspond to times when the accelerometer either ran out of power or was removed from the gauge for recharging.

(Figures 4 and 5 were created using 10 min averages), whereas with a mechanically forced system the analysis can focus only on the immediate response following the perturbation. Finally, data from a naturally forced system can be analyzed only in the frequency domain, whereas data from a mechanically displaced system can be analyzed in either the time or frequency domain, though it should be noted that both analyses give comparable results (as shown in Figure 3). This all said, with high-precision (e.g., 24-bit) accelerometers now becoming widely available, it may be possible to employ passive seismic energizing of the accelerometer, wherein no wind driving is required.

[21] Should mechanical excitation of the gauge be desired (for instance, if the gauge is to be installed in a pit or if the analysis is to utilize the time domain), one efficient and reliable method for perturbing the system is to position an electromagnetic coil next to a ferrous part of the gauge, which can then allow for the coil to be briefly powered at set intervals (an example of this forcing mechanism is presented within the auxiliary material). Furthermore, by measuring the inductance of the coil itself, the relative position of the gauge can be measured through time, obviating the accelerometer and further simplifying the system.

[22] Finally, it should be noted that like all precipitation gauges, this instrument can be subject to errors including rainfall undercatch (due to wind effects), clogging and/or changing readings due to contamination [Sieck et al., 2007], and possible temperature-related noise. To combat rainfall undercatch, the device could be placed into a pit and be mechanically perturbed at specified time intervals using simple piezoelectric motors, solenoids and/or electromagnets, as discussed above. This would have the additional advantage of limiting wind vortex-related resonance modes (though this phenomenon was not observed to be a major influence during field testing). With regard to contamination, since the device has no moving parts, clogging would only be of concern in a drainage system (such as an autosiphon); a well-designed screen or filter could likely prevent most clogging occurrences. For changes in gauge mass due to biological fouling (such as moss or dust), the gauge could be configured to monitor relative changes in mass after each emptying event. Finally, the Young's modulus of the spring will vary slightly with temperature; this effect will likely be minor in the range of temperatures experienced by the gauge, on the order of $0.5-2 \%$ per $100^{\circ} \mathrm{C}$, depending on the material [e.g., Dodge, 1915]. At the same time, monitoring temperature should allow for a straightforward correction. Altogether, these errors should be kept in mind when implementing this system, so as to ensure that they are mitigated or controlled.

\section{Conclusion}

[23] We developed a simple method for measuring both rainfall and evaporation via a single gauge, based on measurements of the natural frequency of the gauge itself, and demonstrated the feasibility of the concept using a prototype. Our configuration had an accuracy of approximately $1 \mathrm{~mm}$; with optimization of the design, installation, and data processing framework, this accuracy can be expected to markedly improve.

[24] As currently configured, the system uses nominal power (it could be powered by a small solar panel in the field) and requires minimal maintenance. It addresses several disadvantages of other rainfall and evaporation measurement devices, provides the prospective ability to be used in windy environments or in ocean-based applications (though 
considerable challenges would need to be addressed in any marine installation), contains no moving parts (which can introduce friction-induced errors or malfunction altogether), and has the potential to be insensitive to wind-induced noise.

[25] To be placed into widespread use, the design will need to be optimized, and features such as an autosiphon for precipitation or a filling system for evaporation measurements may need to be incorporated. Altogether, the proposed system has the potential to provide a low-cost, easy-to-use, and accurate way for research scientists, irrigation managers, and others to monitor cumulative rainfall and evaporation.

[26] Acknowledgments. This work was supported by Oregon State University and the National Science Foundation (award 0943682), who we thank. We also thank Kyle Porth, Kyle Hollis, and Allen Niman for their excellent ideas and suggestions on this project and offer our gratitude to Majdi Abou Najm and John W. Lane for their collaboration. Finally, we would like to acknowledge and sincerely thank the reviewers, whose feedback led to substantial improvement of the manuscript.

\section{References}

Bakkehøi, S., K. Øien, and E. J. Førland (1985), An automatic precipitation gauge based on vibrating-wire strain gauges, Nord. Hydrol., 16, 193-202.

Dodge, H. L. (1915), The change in the elasticity of a mild steel wire with current and external heating, Phys. Rev., 5, 373-384, doi:10.1103/ PhysRev.5.373.

Drexler, J. Z., R. L. Snyder, D. Spano, and K. T. Paw U (2004), A review of models and micrometeorological methods used to estimate wetland evapotranspiration, Hydrol. Processes, 18, 2071-2101, doi:10.1002/ hyp. 1462.

Duchon, C. E. (2008), Using vibrating-wire technology for precipitation measurements, in Precipitation: Advances in Measurement, Estimation and Prediction, edited by S. C. Michaelides, pp. 33-58, Springer, Berlin, doi:10.1007/978-3-540-77655-0_2.

Duchon, C. E., and G. R. Essenberg (2001), Comparative rainfall observations from pit and aboveground rain gauges with and without wind shields, Water Resour. Res., 37(12), 3253-3263, doi:10.1029/2001WR000541.

Dugas, W. A., and W. L. Bland (1989), The accuracy of evaporation measurements from small lysimeters, Agric. For. Meteorol., 46, 119-129, doi:10.1016/0168-1923(89)90116-0.
Grismer, M. E., M. Orang, R. Snyder, and R. Matyac (2002), Pan evaporation to reference evapotranspiration conversion methods, J. Irrig. Drain. Eng., 128, 180-184, doi:10.1061/(ASCE)0733-9437(2002)128:3(180).

Holland, P. W., and R. E. Welsch (1977), Robust regression using iteratively reweighted least-squares, Commun. Stat. Theor. Methods, 6(9), 813-827, doi:10.1080/03610927708827533.

Humphrey, M. D., J. D. Istok, J. Y. Lee, J. A. Hevesi, and A. L. Flint (1997), A new method for automated dynamic calibration of tippingbucket rain gauges, J. Atmos. Oceanic Technol., 14, 1513-1519, doi:10.1175/1520-0426(1997)014<1513:ANMFAD>2.0.CO;2.

Hwang, H. J. (1970), Power density spectrum of surface wind on Palymra Island, Mon. Sci. Rev. Singapore, 98(1), 70-74.

Kuligowski, R. J. (1997), An overview of National Weather Service quantitative precipitation estimates, TDL Off. Note 97-4, NOAA, Silver Spring, Md.

Lathy, B. P. (1998), Signal Processing and Linear Systems, Oxford Univ. Press, New York.

Nayak, A., D. G. Chandler, D. Marks, J. P. McNamara, and M. Seyfried (2008), Correction of electronic record for weighing bucket precipitation gauge measurements, Water Resour. Res., 44, W00D11, doi:10.1029/ 2008WR006875.

Nemani, R. R., and S. W. Running (1989), Estimation of regional surface resistance to evapotranspiration from NDVI and thermal-IR AVHRR data, J. Appl. Meteorol., 28, 276-284, doi:10.1175/1520-0450(1989) 028<0276:EORSRT $>2.0$. CO; 2 .

Nystuen, J. A. (1999), Performance of automatic rain gauges under different rainfall conditions, J. Atmos. Oceanic Technol., 16, 1025-1043, doi:10.1175/1520-0426(1999)016<1025:RPOARG >2.0.CO;2.

Nystuen, J. A., J. R. Proni, P. G. Black, and J. C. Wilkerson (1996), A comparison of automatic rain gauges, J. Atmos. Oceanic Technol., 13, 62-73, doi:10.1175/1520-0426(1996)013<0062:ACOARG >2.0.CO;2.

Sieck, L. C., S. J. Burges, and M. Steiner (2007), Challenges in obtaining reliable measurements of point rainfall, Water Resour. Res., 43, W01420, doi:10.1029/2005WR004519.

Tanner, B. D. (1990), Automated weather stations, Remote Sens. Rev., 5(1), 73-98, doi:10.1080/02757259009532123.

Tattelman, P. (1982), An objective method of measuring surface ice accretion, J. Appl. Meteorol., 21, 599-612, doi:10.1175/1520-0450(1982) 021<0599:AOMFMS $>2.0 . \mathrm{CO} ; 2$.

Upton, G. J. G., and A. R. Rahimi (2003), On-line detection of errors in tipping-bucket raingauges, J. Hydrol., 278(1-4), 197-212, doi:10.1016/ S0022-1694(03)00142-2. 